\title{
Reversal processes and domain wall pinning in polycrystalline Co-nanowires
}

\author{
M. Brands, ${ }^{1}$ R. Wieser, ${ }^{2}$ C. Hassel,,${ }^{1}$ D. Hinzke, ${ }^{2}$ and G. Dumpich ${ }^{1}$ \\ ${ }^{1}$ Experimentalphysik, Universität Duisburg-Essen, 47048 Duisburg, Germany \\ ${ }^{2}$ Theoretische Physik, Universität Duisburg-Essen, 47048 Duisburg, Germany
}

\begin{abstract}
The magnetization reversal process of single Co nanowires is investigated experimentally by magnetoresistance measurements at low temperatures. The results are compared to magnetic force micrographs of the remanent domain configuration. The theoretical expectation for the reversal process is obtained from Monte Carlo simulations. We find that both the width and the thickness of the wires as well as surface scattering play a significant role for the magnetization reversal processes. While thick $(t>15 \mathrm{~nm})$ and narrow nanowires $(w<800 \mathrm{~nm})$ switch by domain wall nucleation at the wire ends followed by wall motion, the magnetization reversal process in wide and/or thin nanowires is achieved by the generation of a multidomain state of the magnetization. It is shown both experimentally as well as theoretically that the wire width dependence of the coercive field can be used to pin a domain wall between two wire parts of different widths.
\end{abstract}

PACS number(s): 75.10.Hk, 75.40.Mg, 75.60.Ch

\section{INTRODUCTION}

It has been shown that magnetoresistance (MR) measurements can be used to investigate the domain structure and the reversal process of ferromagnetic nanostructures. ${ }^{1}$ One thereby exploits the anisotropic magnetoresistance (AMR) which depends on the relative orientation between magnetization and current direction. ${ }^{2}$ It is possible to distinguish between different magnetization reversal processes. ${ }^{3}$ Particularly, detailed investigations have revealed that the magnetization reversal in wider Co nanowires is accomplished by the generation of a multidomain state of the magnetization in the whole wire, while in narrower wires domain walls nucleate at the wire ends and traverse the wire during reversal. ${ }^{4}$ The coercive field in both cases strongly depends on the wire width $w$ and increases as $\frac{1}{w}$ with decreasing wire width. ${ }^{5,6}$

In the present paper we will show by magnetoresistance measurements in combination with Monte Carlo simulations and magnetic force microscopy in which way the switching process in ferromagnetic nanowires is affected by the sample geometry. Therefore, we systematically varied the width, the thickness, and the interface of our Co nanowires. We obtain a phase diagram where two distinctive regions of the magnetization reversal mechanisms can be distinguished. Moreover, we show that even in complex nanowire geometries with lateral constrictions, Monte Carlo simulations reproduce the experimental observations quite well. Exemplary results are shown for the domain wall pinning process in a Co nanowire consisting of two parts with different widths.

\section{EXPERIMENTAL}

The Co nanowires are fabricated by high-resolution electron-beam lithography (HR-EBL) and lift-off technique on Te-doped GaAs substrates with dimensions of $3.9 \times 3.9$ $\times 0.525 \mathrm{~mm}$ and by subsequent electron-beam evaporation of cobalt in an UHV chamber with a base pressure of $p_{B}=1 \times 10^{-8}$ mbar. Some of the wires are in situ capped with a 2-nm-thick platinum layer or a 10-nm-thick layer of carbon to prevent them from oxidation. Further details can be found in Ref. 7. We use specially customized resist systems providing resist masks with strong undercut to obtain wires of highest quality. Structural investigations were performed using secondary electron microscopy (SEM) and transmissionelectron microscopy (TEM) for which arrays of nanowires were also prepared onto $\mathrm{NaCl}$ substrates precoated with a 15 -nm-thick layer of carbon. For widths larger than $130 \mathrm{~nm}$ the wires have only marginal edge roughness with no tear-off edges at all. With slightly reduced quality, wires with widths down to $32 \mathrm{~nm}$ can be prepared. The length of the wires varies between 40 and $200 \mu \mathrm{m}$, whereby the length has no influence on the reversal process. For the magnetoresistance measurements the carbon-covered wires are contacted from below through a thin Pt wire using a complicated three-step EBL process ${ }^{7}$ while a common two-step EBL process is used to attach contact pads consisting of Au to the Pt-capped and uncapped Co nanowires. For typical sample structures, see, for example, Refs. 7 and 8.

Magnetoresistance measurements were carried out via a four-terminal ac technique in a ${ }^{4} \mathrm{He}$ bath cryostat at a temperature of $T=4.2 \mathrm{~K}$. The sample geometry of a nanowire with width $w$, thickness $t$, and length $l$, and the labeling of its axes, is shown in Fig. 1. Magnetic fields of up to $B=5 \mathrm{~T}$ are applied in a direction in the sample plane parallel to the long wire axis (longitudinal), with the electrical current flowing in the same $(x)$ direction. Only small electrical currents ranging between $I=30 \mathrm{nA}$ and $I=3 \mu \mathrm{A}$ were chosen in order to

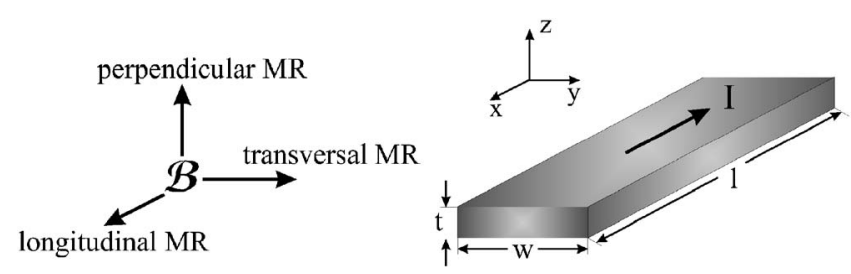

FIG. 1. Schematic picture of a Co nanowire with width $w$, thickness $t$, and length $l$. The current flows in the $x$ direction (longitudinal). The $y$ and $z$ directions are denoted transversal and perpendicular, respectively. 
minimize heating effects. The accuracy of the resistance measurements is of the order of $\frac{\Delta R}{R}=1 \times 10^{-6}$.

Structural investigations reveal that the cobalt has a polycrystalline morphology with an average grain size of $\Phi \approx 7 \pm 2 \mathrm{~nm}$. Electron-diffraction patterns indicate the predominance of hexagonal close-packed (hcp) cobalt with a number of stacking faults.

\section{THEORETICAL}

We consider a classical micromagnetic model ${ }^{9}$ with a cell size of $7 \mathrm{~nm}$, which is of the order of the grain size of our sample. Energy contributions from anisotropy, exchange interaction, driving magnetic field, and dipole-dipole interaction are taken into account. The Hamiltonian is given by

$$
\begin{aligned}
\mathcal{H}= & -J \sum_{\langle i j\rangle} \mathbf{S}_{i} \cdot \mathbf{S}_{j}-d_{a} \sum_{i}\left(\mathbf{e}_{i} \cdot \mathbf{S}_{i}\right)^{2}-\mu_{S} \mathbf{B} \cdot \sum_{i} \mathbf{S}_{i} \\
& -\omega \sum_{i<j} \frac{3\left(\mathbf{S}_{i} \cdot \mathbf{e}_{i j}\right)\left(\mathbf{e}_{i j} \cdot \mathbf{S}_{j}\right)-\mathbf{S}_{i} \cdot \mathbf{S}_{j}}{r_{i j}^{3}},
\end{aligned}
$$

where $\mathbf{S}_{i}=\mu_{i} / \mu_{S}$ are three-dimensional magnetic moments of unit length on a cubic lattice, where $\mu_{S}=M_{S} a^{3}$ denotes the magnetic moment of a cell and $M_{S}=1.43 \times 10^{6} \mathrm{~A} / \mathrm{m}$ the spontaneous magnetization. The cell size is given by $a=7 \mathrm{~nm}$ and corresponds to the experimental findings of the polycrystalline nature of the nanowires with a grain size of about $7 \mathrm{~nm}$.

The first sum is the ferromagnetic exchange between nearest neighbors with coupling constant $J=2 a \mathrm{~A}$ and $A=1.3 \times 10^{-11} \mathrm{~J} / \mathrm{m}$. The second sum is the anisotropy energy given by $d_{a}=K_{a} a^{3}$, with $K_{a}=6.8 \times 10^{5} \mathrm{~J} / \mathrm{m}^{3}{ }^{9 ., 5}$ The direction of this uniaxial anisotropy is constant in amount but random in in-plane direction. The unit vector of the random in-plane direction is given by $\mathbf{e}_{i}$. The third sum is the coupling of the magnetic moments to an external magnetic field $\mathbf{B}$. The last sum is the dipolar interaction, where $\omega=\mu_{0} \mu_{S}^{2} /\left(4 \pi a^{3}\right)$ describes the strength of the dipole-dipole interaction. The unit vectors $\mathbf{e}_{i j}$ point from lattice point $i$ to $j$ and $r_{i j}$ is the distance between these lattice points in units of the lattice constant $a$.

For our simulation we use a Monte Carlo method with a heat-bath algorithm and single-spin-flip dynamics, which is described in detail in Refs. 10 and 11. In order to deal with the long-range dipole-dipole interaction we use a fast-Fourier transformation. ${ }^{12}$ All the simulations are performed at $T=5 \mathrm{~K}$ and start with a configuration where all spins are parallel to the wire.

\section{RESULTS AND DISCUSSION}

The longitudinal magnetoresistance behavior of three platinum-capped Co nanowires with a thickness of $30 \mathrm{~nm}$ cobalt and $2 \mathrm{~nm}$ platinum and widths of $32 \mathrm{~nm}, 120 \mathrm{~nm}$, and $2.16 \mu \mathrm{m}$, respectively, are shown in Fig. 2, where we have plotted the relative resistance $\frac{\Delta R}{R}$ as a function of the magnetic field applied in the $x$ direction. The characteristic MR behavior will be discussed exemplarily for the 103-nm-wide wire. After saturating the nanowire in an external magnetic field of $B=1.5 \mathrm{~T}$ applied longitudinally, the remanent state is

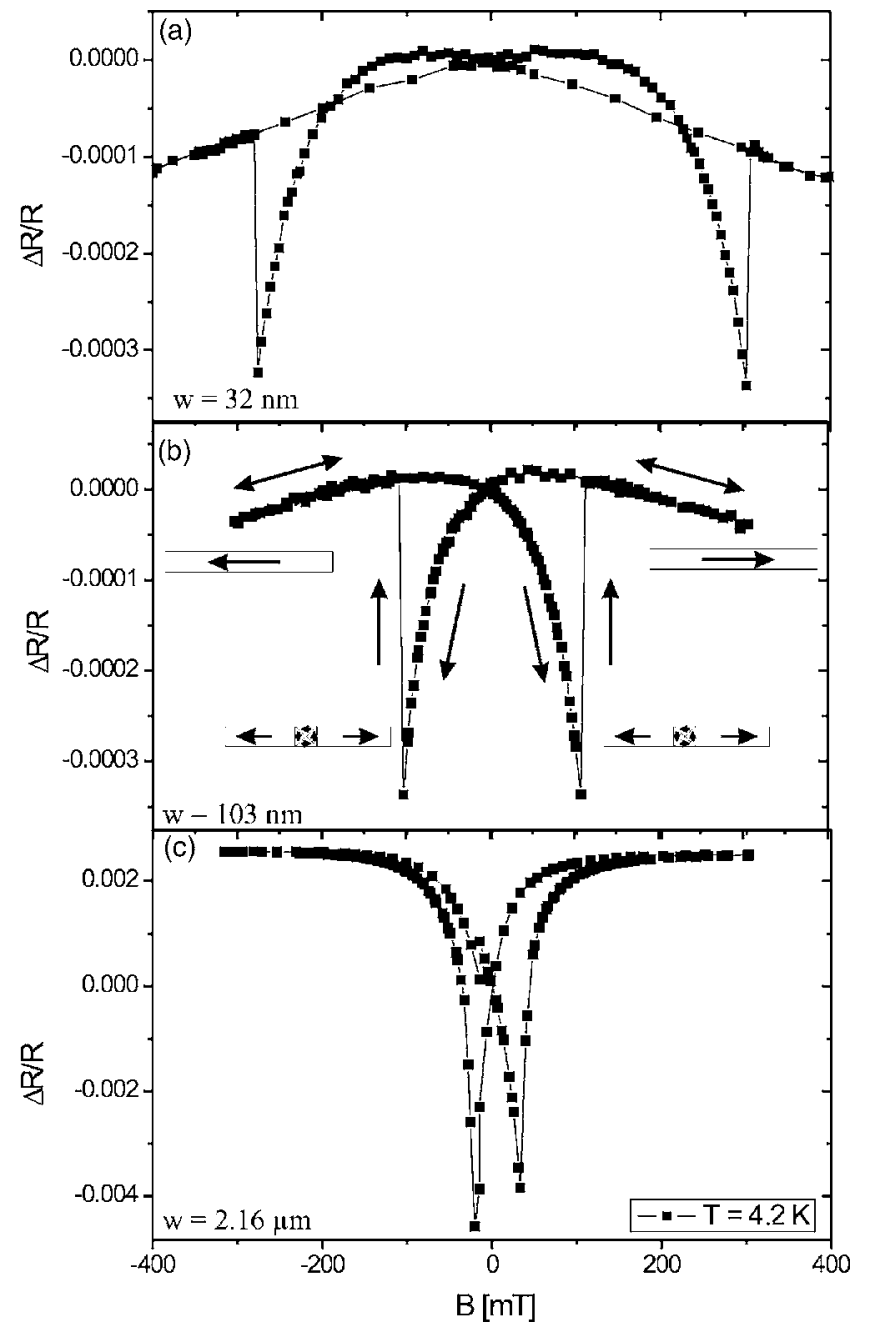

FIG. 2. Longitudinal resistance of Pt-capped Co nanowires as a function of the applied magnetic field. The wires have a uniform thickness of $32 \mathrm{~nm}\left(t_{\mathrm{Pt}}=2 \mathrm{~nm}\right)$ whereas their widths are $32 \mathrm{~nm}$ (a), $103 \mathrm{~nm}$ (b), and $2.16 \mu \mathrm{m}$ (c), respectively. Figure 2(c) was taken from Ref. 4.

monodomainlike. Upon application of a small magnetic field in the reverse direction, a small amount of magnetization components is rotated from a direction along the long wire axis to a transverse direction while simultaneously vortextype domain configurations form at the wire ends. ${ }^{4}$ This leads to a resistance decrease due to AMR. At the coercive field $B_{c} \approx 105 \mathrm{mT}$ the resistance sharply increases and returns to its original value. On the basis of the AMR, this happens when the domain wall is depinned at one or both wire ends and traverses the wire. The same behavior is obtained for the negative field direction. The slightly negative MR in higher fields is due to a small misalignment of the long wire axis and the external magnetic field, which causes a rotation of the magnetization in higher magnetic fields along the direction of the magnetic field instead of the long wire axis. This reduces the resistance according to the AMR effect. We do not observe any contribution due to the classical Lorentz MR. ${ }^{8}$

(a) Width dependence. The reduction of the wire width down to $32 \mathrm{~nm}$ [Fig. 2(a)] does not lead to significant 


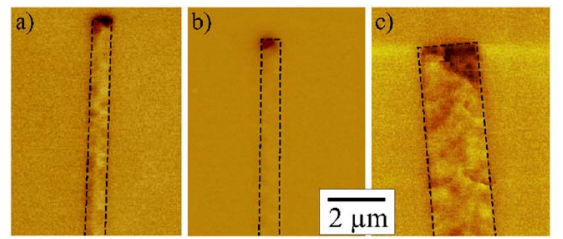

FIG. 3. (Color online) Magnetic force micrographs of three Co nanowires with a thickness of $10 \mathrm{~nm}$ (a) and $30 \mathrm{~nm}$ [(b) and (c)] and widths of $575 \mathrm{~nm}, 545 \mathrm{~nm}$, and $2.1 \mu \mathrm{m}$ (from left to right). The images were taken in remanence after saturation in a field of $B=2 \mathrm{~T}$ applied longitudinally.

deviations from the MR behavior discussed above. The coercive field increases to $B_{c} \approx 305 \mathrm{mT}$ in agreement with our earlier investigations where we were able to show that the coercive fields of Co nanowires obey a $\frac{1}{w}$ law ${ }^{13,6}$ as also has been pointed out by other authors. ${ }^{14,15}$ The resistance maxima in small magnetic fields are due to the destabilization of a ripplelike magnetization configuration, which results from structural inhomogeneities in the smallest wires.

The magnetoresistance of a $2.16-\mu \mathrm{m}$-wide wire is shown in Fig. 2(c). It exhibits two resistance minima at a coercive field of $B_{c} \approx 29 \mathrm{mT}$ as expected for this wire width. ${ }^{4}$ In contrast to the discussion of the two narrower nanowires above, the resistance at $B=0 \mathrm{~T}$ is much lower as compared to its saturation value. This is due to the fact that the remanent state is not monodomainlike, which is typical for wider wires with low aspect ratio. ${ }^{16-18}$ Accordingly, the transversal magnetization components present in remanence lead to a reduction of the resistance due to the AMR. Moreover, the smooth resistance increase for $B>B_{c}$ indicates a magnetization reversal process that incorporates a multidomain state of the magnetization. This is also reflected in the relative resistance decrease at the coercive field $\Delta R\left(B_{c}\right) / \Delta R_{A M R}$ which is a measure for the amount of transversal magnetization components present in the wire at the coercive field. For narrow wires one usually finds values of a few percent (taking into account an AMR effect of the order of $1 \%$ ) since only the domain wall itself and a small amount of transversal magnetization components contribute [see, for example, Figs. 2(a) and 2(b)]. The complex multidomain state of the magnetization in wider wires is reflected in an amount of transversal magnetization, which is about one order of magnitude larger than for a narrow cobalt nanowire [see Fig. 2(c)]. ${ }^{4}$

The remanent state of the magnetization is imaged by magnetic force microscopy (MFM). Figure 3 shows three MFM images of single Co nanowires. Due to their length $l>50 \mu \mathrm{m}$, only a section from the wire ends is shown. The dashed lines indicate the wire dimensions as deduced from the corresponding AFM images (not shown here). Both the wire in the middle (b) and on the right-hand side (c) have a thickness of $30 \mathrm{~nm}$ cobalt (and $10 \mathrm{~nm}$ carbon) whereas their widths are $545 \mathrm{~nm}$ [Fig. 3(b)] and $2.1 \mu \mathrm{m}$ [Fig. 3(c)]. The images were taken in remanence after saturation in a field of $B_{x}=2 \mathrm{~T}$ applied longitudinally. The narrow wire exhibits a single dark spot at its end and absolutely no magnetic contrast everywhere else which corresponds to a single domain state of the magnetization, with the magnetization lying in- (a)
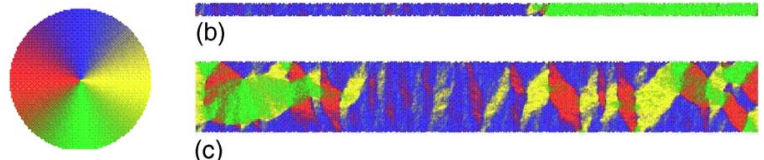

(c)

FIG. 4. (Color online) Snapshots (top view) of simulated Co nanowires close to the coercive field $\mu_{o} H_{c}$ for a thickness of $28 \mathrm{~nm}$ and two different widths, $w=84 \mathrm{~nm}$ and $w=448 \mathrm{~nm}$. The magnetization direction is color coded as blue: initial direction along the wire axis, green: reversed direction; red and yellow: perpendicular in-plane magnetization.

plane and pointing along the long wire axis. ${ }^{19}$ In this case the magnetic stray field only protrudes at the wire ends. Contrarily, the wider wire additionally displays magnetic contrast along the whole wire length, which can be interpreted as a multidomain state of the magnetization. Surprisingly, the smaller but thinner wire [Fig. 3(a)] displays a similar behavior, which will be discussed in Sec. IV C in more detail.

While magnetic force microscopy allows us only to image the remanent state of the Co nanowires, the discussed magnetization reversal processes as a function of the magnetic field and as a function of the wire width are studied in detail by Monte Carlo simulation to gain deeper insight into the reversal mechanisms. Figure 4 shows two snapshots of simulated Co nanowires with a thickness of $t=28 \mathrm{~nm}$ and with two different widths at a magnetic field close to the respective coercive field. The length of the wires is about $4 \mu \mathrm{m}$. For the discussion of the predominant reversal mechanism we choose to present selected snapshots of the wires at the coercive field. Depending on the wire width a crossover between two reversal processes can be found. In the case of wide wires a very complicated domain structure is observed at the coercive field. Here, a large amount of transversal in-plane domains fill up the inner part of the wire and $180^{\circ}$ — as well as $90^{\circ}$ - domain walls are formed. With decreasing wire width a transition is found at a wire width of about $100 \mathrm{~nm}$. In such narrower wires two nuclei originate at one or both ends of the nanowire resulting in in-plane vortex domain walls. At the coercive field $B_{c}$ the domain walls are depinned and traverse the wire within a small magnetic-field interval leading to the reversal of the magnetization. In comparison to the switching process of wider wires, only a small fraction of transversal components of the magnetization are present in the wire during the whole reversal process. This fact can be directly correlated to the MR measurements where one finds a smaller value for the relative resistance decrease at the coercive field in the case of narrow wires. The crossover from a switching process incorporating a multidomain state of the magnetization to one accomplished by domain wall nucleation and traversal depends on the ratio between the exchange interaction and the dipole-dipole interaction [see Eq. (1)].

(b) Interface influence. It is well known that uncapped cobalt nanowires oxidize quickly when exposed to ambient conditions. The cobalt oxide layer on top and at the wire sides is antiferromagnetic and insulating. This changes the magnetization reversal process, which can be seen from Fig. 5(b) where we have plotted the longitudinal MR for a 


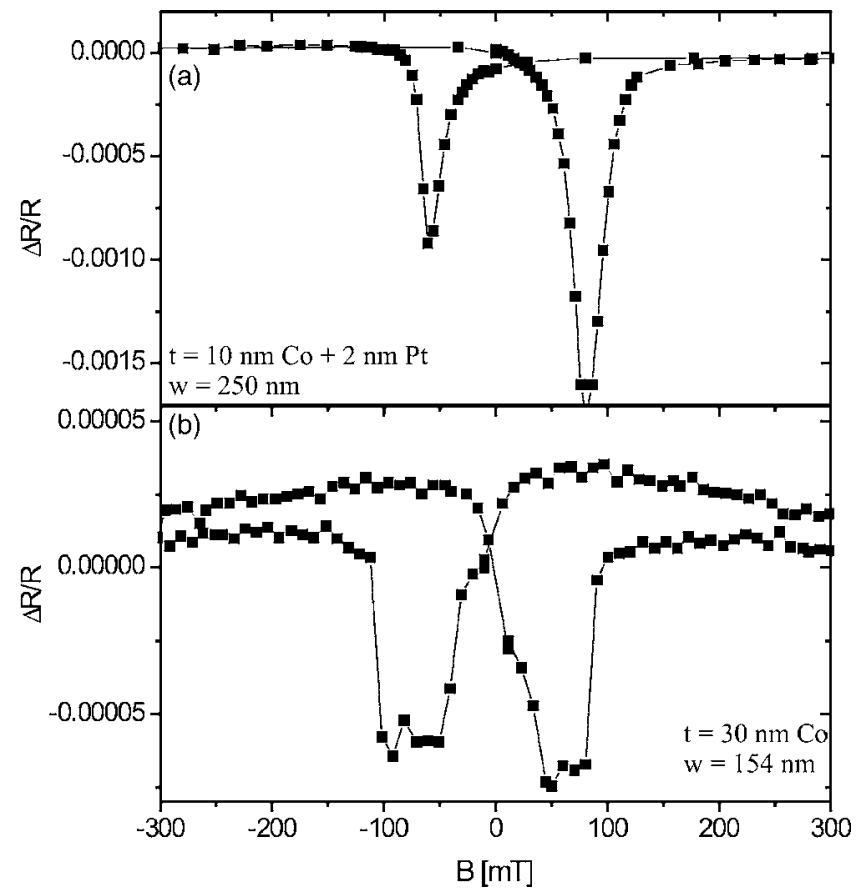

FIG. 5. Longitudinal magnetoresistance of Pt-capped Co nanowire with a width of $250 \mathrm{~nm}$ and a thickness of $10 \mathrm{~nm}$ cobalt and $2 \mathrm{~nm}$ platinum (a) and a 30-nm-thick cobalt nanowire with a width of $154 \mathrm{~nm}$ (b). The bottom graph is taken from Ref. 20.

154-nm-wide uncapped Co nanowire. Qualitatively, the MR shows the same behavior as the capped wires in Fig. 2 and can be explained on the basis of the AMR.

However, while the resistance minima occur in the expected regime and correspond nicely with coercive fields of capped cobalt nanowires, the pronounced plateaus of the resistance are due to domain wall pinning at the $\mathrm{Co} / \mathrm{CoO}$ interface. ${ }^{5}$

(c) Thickness dependence. Figure 5(a) shows the longitudinal magnetoresistance of a 250-nm-wide cobalt nanowire with a thickness of only $10 \mathrm{~nm}$ cobalt and $2 \mathrm{~nm}$ platinum. The resistance in the remanent state is as large as in the saturated state indicating a monodomainlike remanence state. But in contrast to the thicker Co wires, no sharp switching behavior is obtained as has been observed for narrow and thick Co wires [Figs. 2(a) and 2(b)]. The shape of the resistance minimum resembles the MR curve of the widest wire in Fig. 1(c) rather than one of the narrower wires. These measurements indicate that by decreasing the wire thickness a crossover from a magnetization reversal process initiated by domain wall nucleation and traversal to the generation of a multidomain state of the magnetization takes place. The occurrence of such a transition is supported by the observation [see Fig. 3(a)] that for a 10-nm-thin Co nanowire (capped with $10 \mathrm{~nm}$ carbon) with a width of $575 \mathrm{~nm}$ a multidomain state of the magnetization occurs in remanence.

The corresponding Monte Carlo simulations (Fig. 6) display the magnetization reversal behavior of very thin Co wires as discussed above. In agreement with the experimental findings one observes a nucleation process over the whole wire with a large amount of transversal magnetization components. Clearly, the reduction of the dimensionality from

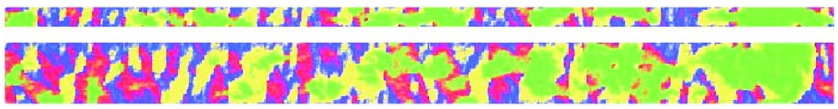

FIG. 6. (Color online) Snapshots (top view) of simulated Co nanowires with thickness $t=7 \mathrm{~nm}$ and two different widths, $w=84 \mathrm{~nm}$ and $w=252 \mathrm{~nm}$. The magnetization direction is color coded as in Fig. 4.

quasi-three-dimensional to quasi-two-dimensional, achieved by the reduction of the wire thickness, affects the local anisotropy and the exchange interaction and leads to domain nucleation and growth over the whole wire.

We compiled the obtained data for Co nanowires in a phase diagram where the type of magnetization reversal process is displayed as a function of the wire width and thickness (Fig. 7). Experimental findings from magnetoresistance measurements are indicated as triangles. Full triangles correspond to a switching incorporating a multidomain state while open triangles represent switching by domain wall nucleation and propagation. Crossed symbols are in the transition region and cannot be unambiguously assigned. Data points from MFM investigations at remanence and Monte Carlo simulations are marked as squares and circles, respectively. Figure 7 clearly reveals that the phase boundary between the two switching mechanisms identified above can be crossed by either a change of the wire thickness or the wire width. Experimental findings correspond reasonably well with the theoretical calculations. The deviation for thicknesses $25 \mathrm{~nm}$ $<t<40 \mathrm{~nm}$ is due to the fact that the simulated nanowires have a length of only about $4 \mu \mathrm{m}$ to allow for reasonable calculation times.

Monte Carlo simulations are also applicable for more complex wire geometries with lateral constrictions. We will

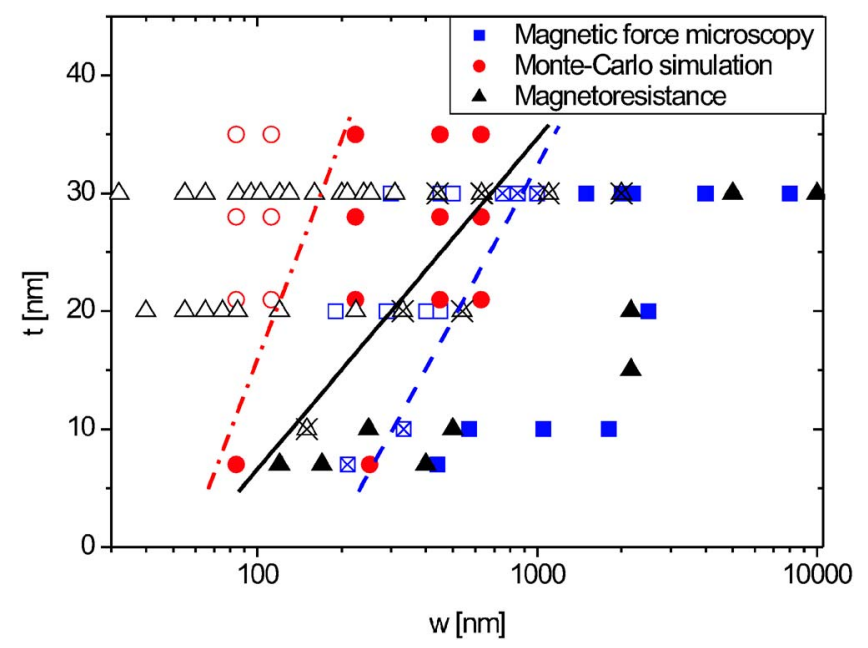

FIG. 7. (Color online) Phase diagram of the switching mechanisms of Co nanowires as a function of their thicknesses and widths. Experimental findings from magnetoresistance measurements (triangles) and MFM imaging (squares) are compared to theoretical values obtained from Monte Carlo simulation (circles). Note that MFM was only performed in remanence. The lines indicate the transition from multidomain switching (full symbols) to a reversal process by domain nucleation and propagation (open symbols). 


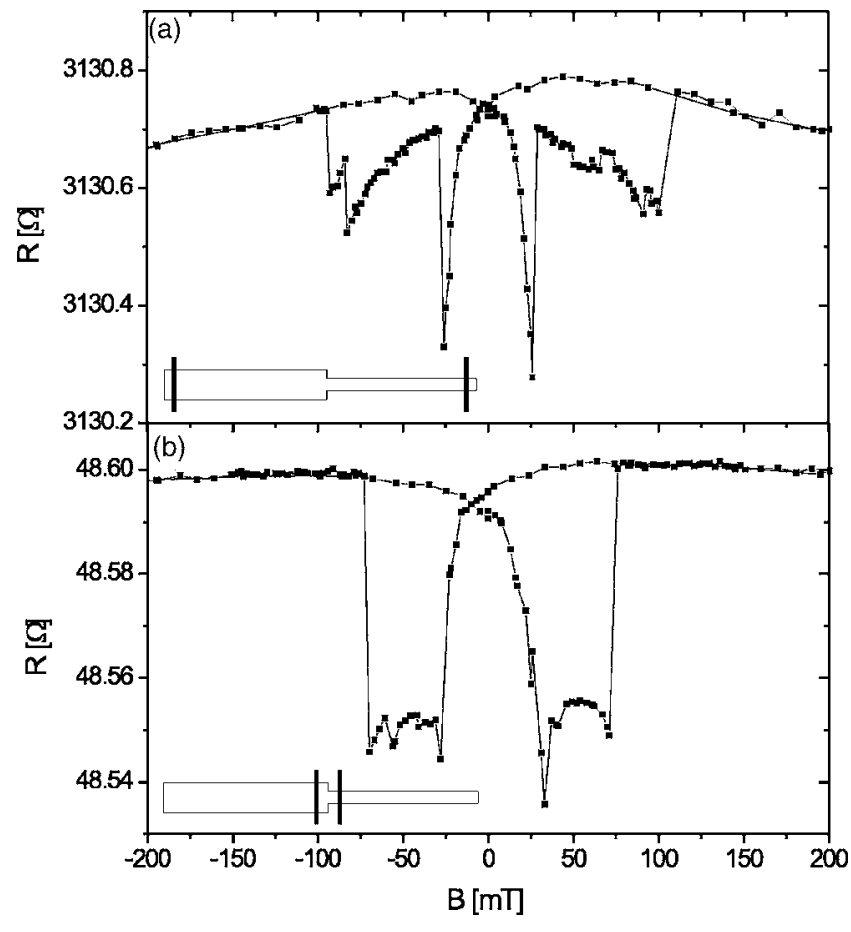

FIG. 8. Longitudinal resistance of a Pt-capped Co nanowire as a function of the applied magnetic field. The wire has a uniform thickness of $32 \mathrm{~nm}\left(t_{\mathrm{Pt}}=2 \mathrm{~nm}\right)$, length $l=100 \mu \mathrm{m}$, and two different widths $w_{1}=85 \mathrm{~nm}, w_{2}=700 \mathrm{~nm}$. The bottom graph (b) shows the resistance for the inner voltage leads of the same wire.

show this exemplarily for a cobalt nanowire, which consists of two parts with different widths. Due to the strong dependence of the coercive field on the wire width, such a system is commonly used to pin a domain wall in the center region between both wire parts. ${ }^{21}$ Figure 8 (a) shows the longitudinal MR of a Pt-capped cobalt wire with a thickness of $30 \mathrm{~nm}$ cobalt and two different wire widths, $85 \mathrm{~nm}$ and $700 \mathrm{~nm}$, respectively. Figure 8(b) shows the MR behavior of the same wire, however, the voltage leads were placed in a distance of only $500 \mathrm{~nm}$ from the center region where the wider and the narrower part of the nanowire merge. The resistance of the Co wire in Fig. 8(a) shows two sharp resistance minima in each field direction, which correspond to two magnetization reversal processes, one for the wider part of the wire at $B_{c} \approx 50 \mathrm{mT}$, the other for the narrower part of the wire at $B_{c} \approx 150 \mathrm{mT}$. In the field range $50 \mathrm{mT}<B_{c}<150 \mathrm{mT} \mathrm{a}$ single domain wall is pinned in the vicinity of the transition region between the wider and the narrower part of the Co wire. To characterize the MR behavior of this part of the wire with higher resolution, we fabricated additional voltage leads, which are placed at a distance of only $500 \mathrm{~nm}$ from the transition region of the wire. The corresponding resistance behavior as measured at these inner voltage leads can be seen in Fig. 8(b). The resistance exhibits two broad minima in the region where the domain wall is located between the inner voltage leads. It is interesting to note that

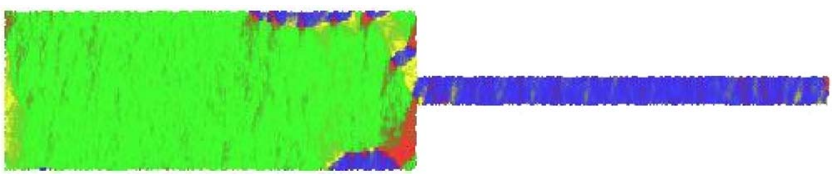

FIG. 9. (Color online) Snapshot (top view) of a simulated Co nanowire between the coercive fields of the wider and the narrower part of the wire. The magnetization direction is as before. The external field is applied along the long wire axis.

domain wall pinning generated by the wire constriction leads to a quite similar MR behavior as has been observed for an oxidized Co wire [Fig. 5(b)] without constrictions, proving that domain wall pinning leads to resistance plateaus.

Concerning the DWR, Fig. 8(b) would naively lead to the interpretation that the DWR is negative. However, as has already been pointed out by other authors, ${ }^{21,22}$ for a system with in-plane magnetization one has to take into account that contributions from the AMR play an important role. We thus checked the domain configuration by Monte Carlo simulations. Applying a longitudinal magnetic field first, the wider and then the narrower part reverse. Between the reversal processes a pinned domain wall at the transition between the two parts can be observed. Figure 9 shows a snapshot of the simulated Co nanowire after the reversal of the wider part. At the transition a vortex structure with $90^{\circ}$ domain walls can be seen. Similar results are obtained in Ref. 23. This large amount of transverse magnetization components in the center region of the wire leads to a negative resistance contribution due to AMR as long as this domain structure is present between the inner voltage leads. Thus, following our discussion in Ref. 8 the negative magnetoresistance can be explained by AMR contributions while the influence of domain wall resistance plays a minor role and cannot be quantified. Note that the Monte Carlo simulation only gives reasonable results if the magnetization configuration is calculated for a fixed external magnetic field in equilibrium, which validates our concept of presenting snapshots of the equilibrium domain structure close to the coercive field.

In conclusion, we have shown that the magnetoresistance behavior of single cobalt nanowires yields a detailed understanding of the magnetization reversal processes of such wires. For this, the MR measurements have to be accompanied by magnetic force microscopy and theoretical simulations of the domain structure and the switching mechanisms. While narrow and thick nanowires exhibit a monodomainlike remanent state and switch by domain wall nucleation and propagation a transition to multidomain state behavior occurs when the wire width is increased or the wire thickness is decreased.

\section{ACKNOWLEDGMENTS}

This work was supported by the Deutsche Forschungsgemeinschaft (Grants No. SFB 491 and No. NO290). 
${ }^{1}$ K. Hong and N. Giordano, J. Magn. Magn. Mater. 151, 396 (1995).

${ }^{2}$ R. McGuire and R. I. Potter, IEEE Trans. Magn. MAG-11, 1018 (1975).

${ }^{3}$ S. J. Blundell, C. Shearwood, M. Gester, M. J. Baird, J. A. C. Bland, and H. Ahmed, J. Appl. Phys. 75, 5249 (1994).

${ }^{4}$ B. Leven and G. Dumpich, Phys. Rev. B 71, 064411 (2005).

${ }^{5}$ B. Hausmanns, T. P. Krome, G. Dumpich, E. F. Wassermann, D. Hinzke, U. Nowak, and K. D. Usadel, J. Magn. Magn. Mater. 240, 297 (2001).

${ }^{6}$ M. Brands, B. Leven, and G. Dumpich, J. Appl. Phys. 97, 114311 (2005).

${ }^{7}$ M. Brands, O. Posth, and G. Dumpich, Superlattices Microstruct. 37, 380 (2005).

${ }^{8}$ M. Brands and G. Dumpich, J. Phys. D 38, 822 (2005).

${ }^{9}$ J. Fidler and T. Schrefl, J. Phys. D 33, R135 (2000).

${ }^{10}$ U. Nowak, in Annual Reviews of Computational Physics IX, edited by D. Stauffer (World Scientific, Singapore, 2001), p. 105.

${ }^{11}$ U. Nowak, R. W. Chantrell, and E. C. Kennedy, Phys. Rev. Lett. 84, 163 (2000).

${ }^{12}$ D. Hinzke and U. Nowak, J. Magn. Magn. Mater. 221, 365
(2000).

${ }^{13}$ B. Hausmanns, T. P. Krome, and G. Dumpich, J. Appl. Phys. 93, 1 (2003).

${ }^{14}$ K. J. Kirk, J. N. Chapman, S. McVitie, P. R. Aitchison, and C. D. W. Wilkinson, Appl. Phys. Lett. 75, 3683 (1999).

${ }^{15}$ S. Y. Yuan, H. N. Bertram, J. F. Smyth, and S. Schultz, IEEE Trans. Magn. 28, 3171 (1992).

${ }^{16}$ E. Seynaeve, G. Rens, A. V. Volodin, K. Temst, and C. van Haesendonk, J. Appl. Phys. 89, 531 (2001).

${ }^{17}$ A. Aharoni, J. Appl. Phys. 63, 5879 (1988).

${ }^{18}$ Y. Shimazu, M. Ohkubo, and K. Morinaga, J. Magn. Magn. Mater. 240, 17 (2002).

${ }^{19}$ U. Ebels, A. Radulescu, Y. Henry, L. Piraux, and K. Ounadjela, Phys. Rev. Lett. 84, 983 (2000).

${ }^{20}$ G. Dumpich, T. P. Krome, and B. Hausmanns, J. Magn. Magn. Mater. 248, 241 (2002).

${ }^{21}$ K. Miyake, T. K. Shigeto, K. Mibu, T. Shinjo, and T. Ono, J. Appl. Phys. 91, 3468 (2002).

${ }^{22}$ S. Lepadatu and Y. B. Xu, Phys. Rev. Lett. 92, 127201 (2004).

${ }^{23}$ W. Y. Lee, A. Hirohata, H. T. Leung, Y. B. Xu, S. M. Gardiner, C. C. Yao, and J. A. C. Bland, IEEE Trans. Magn. 36, 3018 (2000). 